\title{
No Degeneracy of the Ground State for the Impact Parameter Model
}

\author{
Héctor C. Merino ${ }^{1}$, Juan Héctor Arredondo ${ }^{2}$ \\ ${ }^{1}$ Universidad Autónoma de Guerrero, Unidad Académica de Matemáticas, \\ Chilpancingo, México \\ ${ }^{2}$ Departamento de Matemáticas, Universidad Autónoma Metropolitana-Iztapalapa, \\ Distrito Federal, México \\ E-mail:mech@xanum.uam.mx,iva@xanum.uam.mx \\ Received May 25, 2011; revised July 6, 2011; accepted July 13, 2011
}

\begin{abstract}
A time dependent Hamiltonian associated to the impact parameter model for the scattering of a light particle and two heavy ones is considered. Existence and non degeneracy of the ground state is shown.
\end{abstract}

Keywords: Impact Parameter Model, Non Degeneracy of the Ground State

\section{Introduction}

In $[1,2]$, the impact parameter model for the scattering of two heavy particles and a light one is studied, where it is assumed that the heavy particles are infinitely massive and that their motion along a classical trajectory is not affected by the light particle. Also, rigorous proof from first principles of the validity of Massey's criterion is given $[1,3]$.

The above mentioned results were proved for a simple Hamiltonian, by means of an adiabatic argumentation. Now we study a more complicated one than in [1], where a precise knowledge of the discrete spectrum of the corresponding Hamiltonian was needed.

A physical ground state is a state of minimal energy, and therefore it has a relevant role in quantum theories. See for instance [4-17].

In this work we prove non degeneracy of the ground state for the Hamiltonian

$$
H(t)=-\frac{1}{2} \Delta-\lambda_{1} V_{1}-\mu_{1} V_{2}-\lambda_{2} V_{1, \rho}-\mu_{2} V_{2, \rho},
$$

defined as an operator in the Hilbert space $L^{2}\left(\mathbb{R}^{n}\right)$ of all complex valued Lebesgue measurable square integrable functions on $\mathbb{R}^{n}$, with domain $H^{2}\left(\mathbb{R}^{n}\right)$, the Sobolev space of order two [18]. $\Delta$ is the Laplace operator [11].

$$
\Delta=\frac{\partial^{2}}{\partial x_{1}^{2}}+\cdots+\frac{\partial^{2}}{\partial x_{n}^{2}},
$$

with derivatives in the distribution sense, and, $\lambda_{1}, \lambda_{2}, \mu_{1}$, $\mu_{2}$ are positive constants. Also, for $k=1,2$, we will take the potentials $V_{k}$ of rank one:

$$
V_{k} \varphi=\left(g_{k}, \varphi\right) g_{k}, \quad \forall \varphi \in L^{2}\left(\mathbb{R}^{n}\right),
$$

with $g_{1}, g_{2}$ fixed elements in $L^{2}\left(\mathbb{R}^{n}\right)$. Here $(\cdot, \cdot)$ denotes the scalar product in $L^{2}\left(\mathbb{R}^{n}\right)$, antilinear on the factor on the left. Moreover,

$$
V_{\kappa, \rho} \varphi=\left(g_{\kappa, \rho}, \varphi\right) g_{\kappa, \rho}, \quad g_{\kappa, \rho}(x):=g_{\kappa}(x-\rho(t)),
$$

$\rho(t)$ being a continuous function on $\mathbb{R}$ with values in $\mathbb{R}^{n}$ satisfying $\rho(0)=0 \in \mathbb{R}^{n}$ and

$$
\lim _{|t| \rightarrow \infty}|\rho(t)|=\infty
$$

We denote by ${ }^{\wedge}$ the Fourier transform [19], as an unitary operator in $L^{2}\left(\mathbb{R}^{n}\right)$ :

$$
\hat{g}(\boldsymbol{p})=\lim _{K \rightarrow \infty} \int_{|x| \leq K} \mathrm{e}^{-i p x} g(\boldsymbol{x}) \mathrm{d} \boldsymbol{x}, \quad g \in L^{2}\left(\mathbb{R}^{n}\right),
$$

where the limit is taken in the $L^{2}$-norm.

\section{Main Theorem}

From Weyl's theorem [16], one knows that for each $t \in \mathbb{R}, H(t)$ is a self-adjoint operator with discrete spectrum in $(-\infty, 0)$. The eigenvector corresponding to the infimum of the spectrum of $H(t)$ is called the 
ground state for $H(t)$. The following theorem was proved in [20].

Theorem 2.1. For $i=1,2$, let $g_{i} \in L^{2}\left(\mathbb{R}^{n}\right)$ and $\hat{g}_{1}$ nonnegative functions obeying $|\boldsymbol{p}| \hat{g}_{1} \in L^{2}(\mathbb{R})$. Moreover, we suppose the constants $\lambda_{i}, \mu_{i}$ in Equation (1) satisfy

$$
\lambda_{1}>\mu_{1}+\mu_{2}>\mu_{1}>\lambda_{2}>\mu_{2}>0 .
$$

such that $0<E_{0}(2)<E_{1}$ and $0<E_{\lambda_{2}}<E_{\mu_{1}}$. Here $-E_{1},-E_{0}(2),-E_{\lambda_{2}}$, and $-E_{\mu_{1}}$ are the ground state eigenvalues associated to

$$
\begin{gathered}
-\frac{1}{2} \Delta-\lambda_{1} V_{1},-\frac{1}{2} \Delta-\left(\mu_{1}+\mu_{2}\right) V_{2}, \\
-\frac{1}{2} \Delta-\lambda_{2} V_{1},-\frac{1}{2} \Delta-\mu_{1} V_{2},
\end{gathered}
$$

respectively. Then the following statements are valid:

1) The eigenvalue $-E_{0}$, corresponding to the ground state for the operator

$$
H(0)=-\frac{1}{2} \Delta-\left(\lambda_{1}+\lambda_{2}\right) V_{1}-\left(\mu_{1}+\mu_{2}\right) V_{2},
$$

and the eigenvalue $-E_{\infty}$, corresponding to the ground state for the operator

$$
H( \pm \infty)=-\frac{1}{2} \Delta-\lambda_{1} V_{1}-\mu_{1} V_{2},
$$

are strictly negative and the inequality $-E_{0}<-E_{\infty}$ holds.

2) The eigenvale $-E(t)$, corresponding to the ground state for $H(t)$ for all $t \in \mathbb{R}$ lies in the interval $\left[-E_{0},-E_{\infty}\right)$.

3) In the interval $\left(-E_{\infty},-E_{1}\right]$ there are no eigenvalues of $H(t)$ for every $t \in \mathbb{R}$.

We mention that for a given function $0 \neq g \in L^{2}\left(\mathbb{R}^{n}\right)$, one can find a sufficiently large positive constant $\alpha_{0}$ such that the operator

$$
-\frac{1}{2} \Delta-\alpha(g, \cdot) g
$$

has a (unique) negative eigenvalue $-E_{\alpha}$ for $\alpha \geq \alpha_{0}$. In fact, $-E$ is a negative eigenvalue iff [1]

$$
\frac{1}{\alpha}=\left\|\frac{\hat{g}}{\left(\frac{p^{2}}{2}+E\right)^{1 / 2}}\right\|^{2},
$$

where we denote $p^{2}:=|\boldsymbol{p}|^{2}$. Note also that for a given $g$ the right hand side of (5) is a monotone decreasing function of $E$. Therefore, given functions $g_{i}$ in $L^{2}\left(\mathbb{R}^{n}\right)$ one can find constants $\lambda_{i}, \mu_{i}(i=1,2)$ large enough for the hypotheses of the theorem to hold.

We will prove in this manuscript that under the hypotheses of theorem 2.1, for $t \in \mathbb{R}$ the ground state of $H(t)$ is not degenerate.

Let $-E(t)$ be the ground state eigenvalue of the time dependent operator given by Equation (1). We define $\Theta(p):=\frac{p^{2}}{2}+E(t)$ and

$$
a_{i i}=\frac{1}{\lambda_{i}}-\left\|\frac{\hat{g}_{1}}{\sqrt{\Theta}}\right\|^{2} ; d_{i i}=\frac{1}{\mu_{i}}-\left\|\frac{\hat{g}_{2}}{\sqrt{\Theta}}\right\|^{2} \text { for } i=1,2 .
$$

Moreover,

$$
\begin{aligned}
& a_{12}=-\left(\hat{g}_{1}, \Theta^{-1} \hat{g}_{1, \rho}\right), \\
& b_{11}=-\left(\hat{g}_{11}, \Theta^{-1} \hat{g}_{2}\right), \\
& b_{12}=-\left(\hat{g}_{2}, \Theta^{-1} \hat{g}_{1, \rho}\right), \\
& b_{21}=-\left(\hat{g}_{2, \rho}, \Theta^{-1} \hat{g}_{1}\right), \\
& d_{12}=-\left(\hat{g}_{2}, \Theta^{-1} \hat{g}_{2, \rho}\right)
\end{aligned}
$$

Lemma 2.1. Let $-E(t)$ be the ground state eigenvalue of the time dependent operator $H(t)$ given by Equation (1). Then, the matrix equation

$$
\begin{aligned}
& M=\left(\begin{array}{l}
\boldsymbol{x} \\
\boldsymbol{y}
\end{array}\right)=0 \in \mathbb{R}^{4}, \\
& M=\left(\begin{array}{ll}
A & B^{T} \\
B & D
\end{array}\right):=\left\{\begin{array}{llll}
a_{11} & a_{12} & b_{11} & b_{21} \\
a_{12} & a_{22} & b_{12} & b_{11} \\
b_{11} & b_{12} & d_{11} & d_{12} \\
b_{21} & b_{11} & d_{12} & d_{22}
\end{array}\right\},
\end{aligned}
$$

has a nontrivial solution. Furthermore

$$
\operatorname{det}(D) \equiv \operatorname{det}\left[\left(\begin{array}{ll}
d_{11} & d_{12} \\
d_{12} & d_{22}
\end{array}\right)\right]>0 \quad(\forall t \in \mathbb{R}) .
$$

Proof: Let $\psi(t)$ the eigenvector for $H(t)$ with respective eigenvalue $-E(t)$, then the Fourier transform of $\psi(t)$ is given by

$$
\begin{aligned}
\hat{\psi}(t)= & \lambda_{1}\left(g_{1}, \psi\right) \frac{\hat{g}_{1}}{\Theta(\boldsymbol{p})}+\lambda_{2}\left(g_{1, \rho}, \psi\right) \frac{\hat{g}_{1, \rho}}{\Theta(\boldsymbol{p})} \\
& +\mu_{1}\left(g_{2}, \psi\right) \frac{\hat{g}_{2}}{\Theta(\boldsymbol{p})}+\mu_{2}\left(g_{2, \rho}, \psi\right) \frac{\hat{g}_{2, \rho}}{\Theta(\boldsymbol{p})},
\end{aligned}
$$

where $\Theta(\boldsymbol{p}):=\frac{p^{2}}{2}+E(t)$. The Plancherel theorem implies that $(u, v)=(\hat{u}, \hat{v}) \forall u, v \in L^{2}\left(\mathbb{R}^{n}\right)$. Taking inner products in (9) with $\hat{g}_{1}$ and $\hat{g}_{1, \rho}$ for $i=1,2$, we get 


$$
\begin{aligned}
& \left(\hat{g}_{1}, \hat{\psi}\right)=\lambda_{1}\left(\hat{g}_{1}, \hat{\psi}\right)\left\|\frac{\hat{g}_{1}}{\sqrt{\Theta}}\right\|^{2}+\lambda_{2}\left(\hat{g}_{1, \rho}, \hat{\psi}\right)\left(\hat{g}_{1}, \Theta^{-1} \hat{g}_{1, \rho}\right)+\mu_{1}\left(\hat{g}_{2}, \hat{\psi}\right)\left(\hat{g}_{1}, \Theta^{-1} \hat{g}_{2}\right)+\mu_{2}\left(\hat{g}_{2, \rho}, \hat{\psi}\right)\left(\hat{g}_{1}, \Theta^{-1} \hat{g}_{2, \rho}\right), \\
& \left(\hat{g}_{1, \rho}, \hat{\psi}\right)=\lambda_{1}\left(\hat{g}_{1}, \hat{\psi}\right)\left(\hat{g}_{1, \rho}, \Theta^{-1} \hat{g}_{1}\right)+\lambda_{2}\left(\hat{g}_{1, \rho} \hat{\psi}\right)\left\|\frac{\hat{g}_{1, \rho}}{\sqrt{\Theta}}\right\|^{2}+\mu_{1}\left(\hat{g}_{2}, \hat{\psi}\right)\left(\hat{g}_{1, \rho}, \Theta^{-1} \hat{g}_{2}\right)+\mu_{2}\left(\hat{g}_{2, \rho}, \hat{\psi}\right)\left(\hat{g}_{1, \rho}, \Theta^{-1} \hat{g}_{2, \rho}\right), \\
& \left(\hat{g}_{2}, \hat{\psi}\right)=\lambda_{1}\left(\hat{g}_{1}, \hat{\psi}\right)\left(\hat{g}_{2}, \Theta^{-1} \hat{g}_{1}\right)+\lambda_{2}\left(\hat{g}_{1, \rho}, \hat{\psi}\right)\left(\hat{g}_{2}, \Theta^{-1} \hat{g}_{1, \rho}\right)+\mu_{1}\left(\hat{g}_{2}, \hat{\psi}\right)\left\|\frac{\hat{g}_{2}}{\sqrt{\Theta}}\right\|^{2}+\mu_{2}\left(\hat{g}_{2, \rho}, \hat{\psi}\right)\left(\hat{g}_{2}, \Theta^{-1} \hat{g}_{2, \rho}\right), \\
& \left(\hat{g}_{2, \rho}, \hat{\psi}\right)=\lambda_{1}\left(\hat{g}_{1}, \hat{\psi}\right)\left(\hat{g}_{2, \rho}, \Theta^{-1} \hat{g}_{1}\right)+\lambda_{2}\left(\hat{g}_{1, \rho}, \hat{\psi}\right)\left(\hat{g}_{2, \rho}, \Theta^{-1} \hat{g}_{1, \rho}\right)+\mu_{1}\left(\hat{g}_{2}, \hat{\psi}\right)\left(\hat{g}_{2, \rho}, \Theta^{-1} \hat{g}_{2}\right)+\mu_{2}\left(\hat{g}_{2, \rho}, \hat{\psi}\right),\left\|\frac{\hat{g}_{2, \rho}}{\sqrt{\Theta}}\right\|^{2} .
\end{aligned}
$$

This system of equations is represented in matrix form precisely by Equation (8), where

$$
\begin{aligned}
& \boldsymbol{x}=\left(\begin{array}{l}
x_{1} \\
x_{2}
\end{array}\right):=\left(\begin{array}{l}
\lambda_{1}\left(\hat{g}_{1}, \hat{\psi}\right) \\
\lambda_{2}\left(\hat{g}_{1, \rho}, \hat{\psi}\right)
\end{array}\right) ; \\
& \boldsymbol{y}=\left(\begin{array}{l}
y_{1} \\
y_{2}
\end{array}\right):=\left(\begin{array}{l}
\mu_{1}\left(\hat{g}_{2}, \hat{\psi}\right) \\
\mu_{2}\left(\hat{g}_{2, \rho}, \hat{\psi}\right)
\end{array}\right)
\end{aligned}
$$

From Theorem 2.1 we deduce the existence of a nontrivial solution to Equation (8).

Now we fix $E>0$. For every $t \in \mathbb{R}$ let us consider the function,

$$
\begin{aligned}
R_{E}(t)= & \left.\left(\frac{1}{\mu_{1}}-\| \frac{\hat{g}_{2}}{\left(\frac{p^{2}}{2}+E\right)^{1 / 2}}\right)^{2}\right)\left(\frac{1}{\mu_{2}}-\left\|\frac{\hat{g}_{2}}{\left(\frac{p^{2}}{2}+E\right)^{1 / 2}}\right\|^{2}\right) \\
& -\left(\hat{g}_{2}, \frac{\hat{g}_{2, \rho}}{\frac{p^{2}}{2}+E}\right)^{2}
\end{aligned}
$$

and observe that for $E_{0}(2)<E$,

$$
\begin{aligned}
R_{E}(t) & =\frac{1}{\mu_{1} \mu_{2}}-\frac{\mu_{1}+\mu_{2}}{\mu_{1} \mu_{2}}\left\|\frac{\hat{g}_{2}}{\left(\frac{p^{2}}{2}+E\right)^{1 / 2}}\right\|^{2}+\left\|\frac{\hat{g}_{2}}{\left(\frac{p^{2}}{2}+E\right)^{1 / 2}}\right\|^{4}-\left(\hat{g}_{2}, \frac{\hat{g}_{2, \rho}}{\frac{p^{2}}{2}+E}\right)^{2} \geq \frac{1}{\mu_{1} \mu_{2}}-\frac{\mu_{1}+\mu_{2}}{\mu_{1} \mu_{2}}\left\|\frac{\hat{g}_{2}}{\left(\frac{p^{2}}{2}+E\right)^{1 / 2}}\right\|^{2} \\
& =\frac{\mu_{1}+\mu_{2}}{\mu_{1} \mu_{2}}\left\|\frac{1}{\mu_{1}+\mu_{2}}-\right\| \frac{\hat{g}_{2}}{\left(\frac{p^{2}}{2}+E\right)^{1 / 2}} \|^{2}>0 .
\end{aligned}
$$

The last inequality being true because of the remark following Equation (5). Also, we have used the Schwarz inequality and the Fourier transform property $\hat{g}_{2, \rho}(\boldsymbol{p})=e^{-i \rho(t) \cdot \boldsymbol{p}} \hat{g}_{2}(\boldsymbol{p})$. When $-E \equiv-E(t)$ is the eigenvalue for $H(t)$, then the determinant of matrix $D$ in Equation (8) satisfies, $\operatorname{det}(D)=R(E(t))$. Theorem 2.1 states that $-E(t) \in\left[-E_{0},-E_{\infty}\right)$ and $E_{\infty}>E_{1}>E_{0}(2)$. Then, (12) gives $\operatorname{det}(D)>0$.

The main result will be proved by showing that the dimension of the eigenspace associated to the ground sate remains constant over time.

Lema 2.2. The ground state for the operator

$$
H(0)=-\frac{1}{2} \Delta-\left(\lambda_{1}+\lambda_{2}\right) V_{1}-\left(\mu_{1}+\mu_{2}\right) V_{2}
$$

is not degenerate.

Proof: Lemma (2.1) assures that $D^{-1}$ exists. Equation (8) implies,

$$
\boldsymbol{y}=-D^{-1} B \boldsymbol{x}, \quad\left(A-B^{T} D^{-1} B\right) \boldsymbol{x}=\mathbf{0} .
$$

We take $C:=A-B^{T} D^{-1} B$, so that,

$$
C=\left(\begin{array}{ll}
C_{11} & C_{12} \\
C_{12} & C_{22}
\end{array}\right),
$$

where 


$$
\begin{aligned}
& c_{11}=a_{11}-\frac{\left(b_{11}^{2} d_{22}-2 b_{11} b_{21} d_{12}+b_{21}^{2} d_{11}\right)}{\operatorname{det} D}, \\
& c_{12}= \\
& a_{12}-\frac{\left(b_{11} b_{12} d_{22}+b_{11} b_{21} d_{11}-b_{11}^{2} d_{12}-b_{12} b_{21} d_{12}\right)}{\operatorname{det} D}, \\
& c_{22}=a_{22}-\frac{\left(b_{11}^{2} d_{11}-2 b_{11} b_{12} d_{12}+b_{21}^{2} d_{22}\right)}{\operatorname{det} D} .
\end{aligned}
$$

From Theorem 2.1, we know that there exists a nontrivial solution to system (8). Thus $\operatorname{det} C=0$. Accordingly,

$$
C=\left(\begin{array}{cc}
c_{11} & c_{12} \\
k c_{11} & k c_{12}
\end{array}\right),
$$

for some constant $k=k(t)$. Moreover, for $t=0$ the matrix $C=C(t)$ is not null. In fact, for this value of $t$, the following terms simplify

$$
\begin{aligned}
& a_{12}=-\left\|\left(\frac{p^{2}}{2}+E_{0}\right)^{-1 / 2} \hat{g}_{1}\right\|^{2}, \\
& d_{12}=-\left\|\left(\frac{p^{2}}{2}+E_{0}\right)^{-1 / 2} \hat{g}_{2}\right\|^{2}, \\
& b_{21}=b_{12}=b_{11}=-\left(\hat{g}_{1},\left(\frac{p^{2}}{2}+E_{0}\right)^{-1} \hat{g}_{2}\right) .
\end{aligned}
$$

It follows that,

$$
\begin{aligned}
c_{12}= & a_{12}-\frac{b_{11}^{2}\left(d_{11}+d_{22}\right)-2 d_{12} b_{11}}{\operatorname{det} D}=-\left\|\frac{\hat{g}_{1}}{\left(\frac{p^{2}}{2}+E_{0}\right)^{1 / 2}}\right\|^{2} \\
& -\frac{\left(\hat{g}_{1}, \frac{\hat{g}_{2}}{\frac{p^{2}}{2}+E_{0}}\right)^{2}\left(\frac{\mu_{1}+\mu_{2}}{\mu_{1} \mu_{2}}-2\left\|\frac{\hat{g}_{2}}{\left(\frac{p^{2}}{2}+E_{0}\right)^{1 / 2} \|^{2}}\right\|^{2}\right.}{\operatorname{det} D} \\
& -\frac{\|\left(\frac{p^{2}}{2}+E_{0}\right)^{-1 / 2}}{\hat{g}_{2} \|^{2}\left(\hat{g}_{1},\left(\frac{p^{2}}{2}+E_{0}\right)^{-1} \hat{g}_{2}\right)^{2}} \\
< & 0, \quad \operatorname{det} D
\end{aligned}
$$

where we use equation (5), the hypothesis $E_{0}(2)<E_{1}$ and statement (3) of theorem 2.1. Therefore,

$$
\left\|\left(\frac{p^{2}}{2}+E_{0}\right)^{-1 / 2} \hat{g}_{2}\right\|^{2}<\frac{\mu_{1}+\mu_{2}}{2 \mu_{1} \mu_{2}} .
$$

Equations (11)-(16) imply,

$$
\begin{aligned}
& \mu_{1}\left(\hat{g}_{2}, \hat{\psi}\right)=\left(\frac{b_{21} d_{12}-b_{11} d_{22}}{\operatorname{det} D}+\frac{c_{11}\left(b_{12} d_{22}-b_{22} d_{12}\right)}{c_{12} \operatorname{det} D}\right) x_{1} \\
& \mu_{2}\left(\hat{g}_{2, \rho}, \hat{\psi}\right)=\left(\frac{b_{11} d_{12}-b_{21} d_{11}}{\operatorname{det} D}+\frac{c_{11}\left(b_{22} d_{11}-b_{12} d_{12}\right)}{c_{12} \operatorname{det} D}\right) x_{1}
\end{aligned}
$$

Substitution of these equalities in Equation (9) gives,

$$
\begin{aligned}
\hat{\psi}(0)= & x_{1}\left(\frac{\hat{g}_{1}}{\frac{p^{2}}{2}+E_{0}}+k_{1} \frac{\hat{g}_{1, \rho}}{\frac{p^{2}}{2}+E_{0}}\right. \\
& \left.+k_{2} \frac{\hat{g}_{2}}{\frac{p^{2}}{2}+E_{0}}+k_{3} \frac{\hat{g}_{2, \rho}}{\frac{p^{2}}{2}+E_{0}}\right) .
\end{aligned}
$$

Here,

$$
\begin{aligned}
& k_{1}=-\frac{c_{11}}{c_{12}} \\
& k_{2}=\frac{\left(c_{12} b_{21} d_{12}+c_{11} b_{12} d_{22}\right)-\left(c_{12} b_{11} d_{22}+c_{11} b_{22} d_{12}\right)}{c_{12} \operatorname{det} D} \\
& k_{3}=\frac{\left(c_{12} b_{11} d_{12}+c_{11} b_{22} d_{11}\right)-\left(c_{12} b_{21} d_{11}+c_{11} b_{12} d_{12}\right)}{c_{12} \operatorname{det} D} .
\end{aligned}
$$

This determines the vector $\hat{\psi}(0)$ up to a multiplicative constant, and from the Plancherel theorem, also the eigenspace associated to the ground state for $H(0)$, proving the statement of the lemma.

Theorem 2.2. Let $H(t)$ be defined by Equation (1) and suppose the hypotheses of theorem 2.1 hold true. Moreover, we take the curve $\rho: \mathbb{R} \rightarrow \mathbb{R}^{n}$ so that $\rho(t)=a+v t, \quad \forall|t| \geq M$, for some positive constant $M$ and fixed vectors $a, v \in \mathbb{R}^{n}$. Then the dimension of the spectral projection onto the interval $\left[-E_{0},-E_{\infty}\right]$, associated with the selfadjoint operator $H(t)$, is equal to one for each $t \in \mathbb{R}$.

Proof: The resolvent $R_{i}(A)$ of a self-adjoint operator $A$ at $i \in \mathbb{C}$ is defined by $(i I-A)^{-1}$ with $I$ denoting the identity operator on $L^{2}\left(\mathbb{R}^{n}\right)$. We take $H_{2}=H\left(t_{2}\right)$, $H_{1}=H\left(t_{1}\right)$, for two distinct values $t_{1}$ and $t_{2}$ and calculate the difference $R_{i}\left(H_{2}\right)-R_{i}\left(H_{1}\right)$.

$$
\begin{aligned}
R_{i}\left(H_{2}\right) & -R_{i}\left(H_{1}\right)=R_{i}\left(H_{2}\right)\left(H_{2}-H_{1}\right) R_{i}\left(H_{1}\right) \\
& =\lambda_{2} R_{i}\left(H_{2}\right)\left(V_{1, \rho_{1}}-V_{1, \rho_{2}}\right) R_{i}\left(H_{1}\right) \\
& +\mu_{2} R_{i}\left(H_{2}\right)\left(V_{2, \rho_{1}}-V_{2, \rho_{2}}\right) R_{i}\left(H_{1}\right)
\end{aligned}
$$


Here $V_{1, \rho_{1}}$ is given as in Equation (3) with

$g_{i, \rho}(x)=g_{i}(x-\rho(t))$ replaced with

$g_{1, \rho_{1}}(\boldsymbol{x})=g_{1}\left(\boldsymbol{x}-\rho\left(t_{1}\right)\right)$. Also $V_{1, \rho_{2}}, V_{2, \rho_{1}}$, and $V_{2, \rho_{2}}$

being defined similarly. It follows from Equation (1) and standard arguments that

$$
\left\|R_{i}\left(H_{2}\right)-R_{i}\left(H_{1}\right)\right\| \leq \Upsilon\left|t_{2}-t_{1}\right|,
$$

where $\Upsilon$ is a constant uniform in $t_{1}, t_{2} \in \mathbb{R} /[-M, M]$ depending on $\left\||\boldsymbol{p}| g_{\ell}\right\|$ and $\left\|g_{\ell}\right\|, \quad \ell=1,2$. This implies that $R_{i}(H(t))$ is uniformly continuous on $\mathbb{R}$ with respect to the norm topology. Let $P_{S}(B)$ denote the spectral projection of a self-adjoint operator $B$ corresponding to the Borel set $S \subseteq \mathbb{R}$. By functional calculus, we get

$$
P_{\left[-E_{0},-E \infty\right]}\left(H\left(t_{2}\right)\right) \rightarrow P_{\left[-E_{0},-E \infty\right]}\left(H\left(t_{1}\right)\right) \text { as } t_{2} \rightarrow t_{1},
$$

in the operator norm. Therefore, by standard arguments

$$
\operatorname{dim} P_{\left[-E_{0},-E \infty\right]}\left(H\left(t_{2}\right)\right)=\operatorname{dim} P_{\left[-E_{0},-E \infty\right]}\left(H\left(t_{1}\right)\right),
$$

For $t_{2}$ close enough to $t_{1}$. It follows from lemma 2.2 that

$$
\operatorname{dim} P_{\left[-E_{0},-E_{\infty}\right]}(H(t))=1 \quad(\forall t \in \mathbb{R}) .
$$

Remark: We mention that the hypothesis for the curve $\rho(t)$ can be relaxed to the condition that $\rho(t)$ is asymptotic to a straight line.

\section{References}

[1] J. H. Arredondo, “Asymptotic Transition Probabilities," Journal of Mathematical Physics, Vol. 30, No. 10, 1989, pp. 2291-2296. doi:10.1063/1.528558

[2] J. H. Arredondo, "Adiabatic Approximation on the Impact-Parameter Model,” Few-Body Systems, Vol. 10, No. 2, 1991, pp. 59-72. doi:10.1007/BF01352402

[3] H. S. W. Massey, "Collision between Atoms and Molecules at Ordinary Temperatures," Reports on Progress in Physics, Vol. 12, No. 1, 1949, p. 248. doi:10.1088/0034-4885/12/1/311

[4] D. Aharonov, W. van Dam, J. Kempe, Z. Landau, S. Lloyd and O. Regev, "Adiabatic Quantum Computation Is Equivalent to Standard Quantum Computation," 45th Annual IEEE Symposium on Foundations of Computer Science (FOCS'04), Rome, 17-19 October 2004.

[5] F. Hiroshima, "Topics in the Theory of Schrödinger Operators,” World Scientific Publishing Co. Pte. Ltd., Singapore, 2004.
[6] K. Chadan and P. C. Sabatier. "Inverse Problems in Quantum Scattering Theory," Springer-Verlag, New York, 1989.

[7] T. Cubel, B. K. Teo, V. S. Malinoysky, J. R. Guest, A. Reinhard, B. Knuffman, P. R. Berman and G. Raithel, "Coherent Population Transfer of Ground-State Atoms into Rydberg States,” Physical Review A, Vol. 72, 2005, pp. 023405 (1-4).

[8] H. L. Cycon, R. G. Froese, W. Kirsh and B. Simon. "Schrödinger Operators with Application to Quantum Mechanics and Global Geometry,” Springer-Verlag, New York, 1987.

[9] E. Farhi, J. Goldstone, S. Gutmann and M. Sipser, “Quantum Computation by Adiabatic Evolution,” 2000. arXiv:quant-ph/0001106v1

[10] P. D. Hislop and I. M. Sigal, "Introduction to Spectral Theory with Applications to Schrödinger Operators," Springer-Verlag, New York, 1996.

[11] T. Kato, "Perturbation Theory for Linear Operators," Springer-Verlag, Berlin and New York, 1984.

[12] J. O. Lee and J. Yin, "A Lower Bound on the Ground State Energy of Dilute Bose Gas,” Journal of Mathematical Physics, Vol. 51, No. 5, 2010, pp. 053302 (1-31).

[13] E. L. Lieb, R. Seiringer, J. P. Solovej and J. Yngvason, "The Ground State of the Bose Gas," 2003. arXiv:math-ph/0204027v2

[14] H. E. Puthoff, "Ground State of Hydrogen as a ZeroPoint-Fluctuation-Determined State,” Physical Review D, Vol. 35, No. 10, 1987, pp. 3266-3269. doi:10.1103/PhysRevD.35.3266

[15] M. Reed and B. Simon, "Methods of Modern Mathematical Physics, Vol. IV,” Academic Press, New York, 1975.

[16] W. Thirring, "A Course in Mathematical Physics. Vol. III,” Springer-Verlag, New York, 1981.

[17] I. Wilson-Rae, N. Nooshi, W. Zwerger and T. J. Kippenberg, "Theory of Ground State Cooling of a Mechanical Oscillator Using Dynamical Backaction,” Physical Review Letters, Vol. 99, No. 9, 2007, Article ID: 093901 (1-4).

[18] P. D. Lax, “Functional Analysis,” John Wiley \& Sons Inc., New York, 2002.

[19] M. Reed and B. Simon, "Methods of Modern Mathematical Physics, Vol. II,” Academic Press, New York, 1975.

[20] J. H. Arredondo and P. Seibert, "Characterization of the Fundamental State Eigenvalue for Some Time Dependent Hamiltonians,” Aportaciones Matemáticas. Serie Comunicaciones, Vol. 29, No. 3, 2001, pp. 3-10. 